\title{
Selenium Inhibits Homocysteine-Induced Endothelial Dysfunction and Apoptosis via Activation of AKT
}

\author{
Hui Ren ${ }^{a, b}$ Jianjun $\mathrm{Mu}^{\mathrm{a}}$ Jingjing $\mathrm{Ma}^{\mathrm{b}}$ Jie Gong ${ }^{\mathrm{b}}$ Jing $\mathrm{Li}^{\mathrm{b}} \quad$ Jiawen Wang ${ }^{\mathrm{b}}$ \\ Tianlin Gao ${ }^{b}$ Peng Zhu ${ }^{b}$ Shikang Zheng ${ }^{b}$ Jing Xie ${ }^{b}$ Bo Yuan ${ }^{b}$ \\ aDepartment of Cardiology, the First Affiliated Hospital of Xi'an Jiaotong University, Xi'an, \\ bDepartment of Cardiology, An Kang City Central Hospital, An Kang, China
}

\section{Key Words}

Cardiovascular disease $\bullet$ Endothelial cell $\bullet$ Homocysteine $\bullet$ Selenium $•$ Apoptosis

\begin{abstract}
Background/Aims: Endothelial cells are crucial in vascular homeostasis. Dysfunction of endothelial cells is involved in the development of cardiovascular diseases (CVD). High plasma homocysteine (Hcy) correlates with CVD while selenium supplementation counteracts development of CVD. However, the underlying mechanism remained unclear. Here, we investigated the effects of selenium on homocysteine-induced endothelial dysfunction. Methods: An animal model of Hcy-induced endothelial dysfunction was established by intragastric administration of L-methionine. Plasma NO and von Willebrand factor (vWF) were quantified using NO assay and ELISA kit respectively. Relaxation was measured in thoracic aortic ring assays. Cell viability and migration were detected by Cell Counting Kit- 8 and BioCoat cell migration chambers respectively. Cellular apoptosis was determined by Annexin V-FITC apoptosis kit. Results: Selenium prevented homocysteine-induced endothelial injury and impairment of endothelium-dependent relaxation. Selenium reversed the impaired viability and migration of endothelial cells induced by homocysteine in a dose-dependent manner. Selenium inhibited the apoptosis of endothelial cells induced by homocysteine, through downregulating of Caspase-3 activity and expression of Caspase- 3 and Bax, and by stimulating $\mathrm{BCl}-2$ expression. Selenium reversed the homocysteine-induced reduction of NO release, and increased the expression and phosphoylation of endothelial nitric oxide synthetase (eNOS) in a dose-dependent manner. Moreover, selenium enhanced AKT phosphorylation, and selenium-induced phosphorylation and expression of eNOS were inhibited by AKT inhibition. NO production, cell viability and migration rescued by selenium were inhibited, while cell apoptosis was reversed by AKT inhibition. Conclusion: Selenium protected against homocysteine-induced dysfunction and apoptosis of endothelial cells through AKT pathway. The observations may provide novel therapeutic opportunities in the treatment of CVD.
\end{abstract}




\section{Cellular Physiology Cell Physiol Biochem 2016;38:871-882

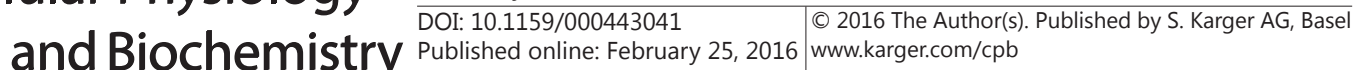 \\ Ren et al.: Selenium Protects Endothelial Function}

\section{Introduction}

Cardiovascular diseases (CVD) are the leading causes of death globally, resulting in approximately 17 million deaths annually [1]. Coronary artery disease and stroke account for about $80 \%$ of CVD deaths [2]. In the United States, $80 \%$ of people aged above 60 are affected by CVD, whose life qualities are severely compromised [3].

A variety of cardiovascular pathological conditions such as hyperlipidemia and hypertension impair the function and structural integrity of endothelium, leading to a cascade of events of atherosclerosis, which in turn worsens the cardiovascular pathological conditions and causes a vicious loop [4]. Endothelial cells have important roles in normal physiological processes including the secretion of chemokines and cell adhesion factors, regulating vascular relaxation and contraction, and promoting angiogenesis [5]. The function and structural integrity of endothelial cells are the most important factors that affecting the development of CVD [6].

Homocysteine (Hcy) is an intermediate metabolite in the metabolic pathway of cysteine and methionine [7]. Elevated levels of plasma Hcy are independent risk factors of CVD, such as coronary heart disease, hypertension and stroke, and Hcy contributes to the development of CVD as a result of endothelial dysfunction [8]. Patients with moderate hyperhomocysteinemia have decreased numbers of endothelial cells with impaired activities [9]. Hcy induces apoptosis, and impairs the proliferation, migration, adhesion of endothelial cells $[10,11]$.

Selenium (Se) is a main ingredient of the glutathione peroxidase which protects cells from the oxidative damage resulting from reactive oxygen species (ROS) [12]. Se has diverse functions including immunogenic, anticarcinogenic, antimutagenic, cell proliferation control and anti-aging activities [13]. Many studies have indicated the benefits that Se may have on patients with hypertension and atherothrombotic diseases [13]. However, until now there is no report regarding the role of Se in the Hcy-induced dysfunction of endothelial cells.

To investigate the effect of Se on Hcy-induced dysfunction of endothelial cells, and reveal the underlying mechanism that Se benefits CVD patients, we performed the present study. We found that Se prevented Hcy-induced endothelia injury and impairment of endotheliumdependent relaxation. Se inhibited Hcy-induced impaired proliferation and migration of human umbilical vein endothelial cells (HUVECs). Se alleviated Hcy-induced apoptosis of HUVECs. Furthermore, the downregulation of NO and eNOS caused by Hcy were rescued by Se treatment. Se increased the phosphorylation of AKT, and Se-induced phosphorylation and expression of eNOS, NO production, cell viability and migration were blocked by AKT inhibition.

\section{Materials and Methods}

Establishment of Hcy-induced endothelial dysfunction animal model

The animal experiment was approved by the Animal Care and Use Committee of Xi'an Jiaotong University. Male Sprague-Dawley rats (150 - $180 \mathrm{~g}$ ) were kept in a room maintained at $24^{\circ} \mathrm{C}$ with a 12 -h light/dark cycle and fed standard rat chow. Rats were randomized into 3 groups: control, Hcy model, and Hcy+Se. Rats in Hcy group and Hcy+Se group were intragastrically administrated L-methionine by $1 \mathrm{~g} / \mathrm{kg} /$ day for 4 weeks [14]. Succinylsulfathiazole (SST) $(0.5 \mathrm{~g} / \mathrm{kg} /$ day) was added to the drink of rats to avoid bacterial proliferation and subsequent folate production. Rats in Hcy+Se group was administered Se (2 g/ $\mathrm{kg}$ /day) since 1 week before. Control rats were administered the same volume of purified water.

\section{Plasma NO and vWF analysis}

Rats were fasted overnight. The next morning rats were anesthetized with sodium pentobarbital (50 $\mathrm{mg} / \mathrm{kg}$, i.p.), and the blood was collected and centrifuged at $3000 \mathrm{rpm}$ for $30 \mathrm{~min}$ at $4^{\circ} \mathrm{C}$ to separate the plasma. The plasma NO levels were assayed by a NO Assay Kit (Nanjing Jiancheng Institute of Biological Engineering, China) and the plasma vWF levels were measured by ELISA (Shanghai Guangrui biotechnology, China). 


\section{Cellular Physiology Cell Physiol Biochem 2016;38:871-882 and Biochemistry Published online: February 25, $2016 \mid \begin{aligned} & \text { (c) } 2016 \text { The Author(s). Published by S. Karger AG, Basel } \\ & \text { www.karger.com/cpb }\end{aligned}$ \\ Ren et al.: Selenium Protects Endothelial Function}

\section{Thoracic aortic ring relaxation assay}

After collecting the blood, the thoracic aortas were isolated immediately. The fat and connective tissue were carefully excised, and the thoracic aortas were cut into rings about $3 \mathrm{~mm}$ long and hung on stainless steel hooklets in improved Krebs solution bubbled with $95 \% \mathrm{O}_{2}$ and $5 \% \mathrm{CO}_{2}$ at $37^{\circ} \mathrm{C}$. Rings were connected to transducers and the Powerlab system (AD instruments, USA). The rings were equilibrated for $2 \mathrm{~h}$ and the Krebs solution was changed every $30 \mathrm{~min}$. The contraction signals were recorded by the Powerlab system and analyzed with the Chart 5 for Windows software (AD instruments, USA). The rings were soaked in $5 \mathrm{~mL}$ Krebs solution supplemented with norepinephrine $\left(10^{-6} \mathrm{M}\right)$. When the vasoconstriction curves reached the plateau phase, acetylcholine $\left(10^{-10}-10^{-6} \mathrm{M}\right), \mathrm{A} 23187\left(10^{-5} \mathrm{M}\right)$ and sodium nitroprusside (10${ }^{9}-10^{-6} \mathrm{M}$ ) were added to observe the endothelium-dependent and independent relaxation of aortic rings respectively. The contraction tension was recorded and relaxation was calculated as the percent reduction from norepinephrine-induced tension.

\section{Isolation and Culture of HUVECS}

This study was approved by the Ethical Committee of Xi'an Jiaotong University and the providers of umbilical cords. Human umbilical vein endothelial cells (HUVECs) were harvested from fresh umbilical cords according to the previous report [15]. Briefly, fresh umbilical veins were digested with $0.1 \%(\mathrm{w} / \mathrm{v})$ collagenase type IV solution (Invitrogen, USA) at $37^{\circ} \mathrm{C}$ for $15 \mathrm{~min}$ to release endothelial cells from the vessel walls. Then, the vessels incubated with collagenase type IV were rinsed with ECM (ScienCell, San Diego, USA). HUVECs were collected by centrifugation at $1000 \mathrm{rpm}$ for $5 \mathrm{~min}$ and cultured in ECM. HUVECs were used at passage 3 .

\section{Cell viability assay}

Cell counting Kit-8 (CCK8, Dojindo Laboratories, Kumamoto) assay was used to assess cell viabilities. Cells were digested with $0.25 \%$ trypsin, and cultured in 96 -well culture plate $\left(1 \times 10^{4}\right.$ cells/well $)$. After different treatment, CCK8 reagent was added into each well, and the cells were incubated at $37^{\circ} \mathrm{C}$ for $2 \mathrm{~h}$. Cell viabilities were measured through absorbance (optical density) by a spectrophotometer at $450 \mathrm{~nm}$.

\section{Cell migration assay}

Cell migration was evaluated using a Bio-Coat cell migration chambers (BD Biosciences, MA, USA), which consist of a 24-well companion plate with cell culture inserts containing a filter with $8 \mu$ m-diameter pores. Briefly, $2 \times 10^{4}$ cell resuspended in $500 \mu \mathrm{L}$ of EGM- 2 medium (Gibco, USA) were seeded in the upper chamber, then vascular endothelial growth factor (VEGF) in EGM-2 medium at $100 \mathrm{mg} / \mathrm{L}$ was placed in the lower compartment of the chamber. After incubation for $24 \mathrm{~h}$, the upper surface of the membrane was wiped with a cotton-tipped applicator to remove non-migrating cells, the migrating cells on the lower surface were fixed with $2 \%$ paraformaldehyde and stained with Giemsa solution. Migrating cells were counted manually in three random microscopic fields.

\section{Cell apoptosis assay}

Cells apoptosis was quantified by the Annexin V-FITC Apoptosis Detection Kit (GenScript, NJ, USA) according to the manufacturer's instruction. Briefly, cells were harvested and washed with PBS. Then the cells were stained with annexin V-FITC staining solution at room temperature for $40 \mathrm{~min}$, before being stained with propidium iodide (PI). The annexin V-FITC bindings at early apoptosis were detected by FITC signal detector $(E x=488 \mathrm{~nm} ; \mathrm{Em}=530 \mathrm{~nm})$, and the PI staining at later stages of apoptosis were detected by the phycoerythrin emission signal detector $(\mathrm{Ex}=488 \mathrm{~nm}$; $\mathrm{Em}=620 \mathrm{~nm})$. The percentages of apoptotic cells were determined by flow cytometer (Becton Dickinson). Results were analyzed by BD FACS diva software.

\section{Western blot analysis}

Total proteins were extracted from cell lysates using RIPA buffer containing phosphatase inhibitor cocktail (Sigma, MO USA), and quantified using the Quick Start Bradford Protein Assay kit (Bio-Rad, Hercules, CA, USA). Proteins were separated by electrophoresis on sodium dodecyl sulfate -polyacrylamide gel electrophoresis (SDS-PAGE) gels and then transferred onto nitrocellulose membranes (Pall Life Science, NY, USA). After being blocked with 5\% non-fat milk at room temperature for $1 \mathrm{~h}$, the membranes were probed with primary antibody (antibodies against eNOS, eNOS phosphoserine 1177, Akt, Akt phosphoserine 473 


\section{Cellular Physiology Cell Physiol Biochem 2016;38:871-882

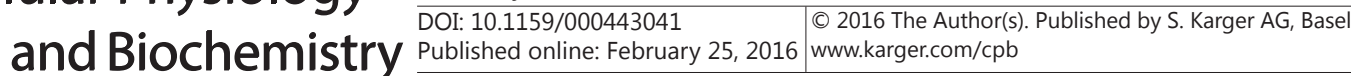 \\ Ren et al.: Selenium Protects Endothelial Function}

respectively, 1:1000 dilution, Cell Signaling Technology) overnight followed by incubation with horse radish peroxidase (HRP) conjugated secondary antibody for $1 \mathrm{~h}$. Immunoblots of protein bands were visualized with an enhanced chemiluminescence (ECL) detection kit (Amersham). The signals were quantified by scanning densitometry.

\section{Measurement of NO production}

NO production was determined by measuring the concentration of nitrite, a metabolite of NO, using a modified Griess reaction method (Nanjing Jiancheng Institute of Biological Engineering, China) according to the manufacturer's instructions. Briefly, supernatant was collected and mixed with an equal volume of modified Griess reagent for the colorimetric assay. After $10 \mathrm{~min}$ of incubation at room temperature, the concentration of the resultant chromophore was measured spectrophotometrically at $550 \mathrm{~nm}$. The nitrite concentration was calculated from nitrite standard curves made from sodium nitrite using the same culture medium.

\section{Statistical analysis}

The obtained data were presented as a mean \pm standard deviation (SD) for a replicate experiment. One-way ANOVA were used for statistical analysis and $\mathrm{P}<0.05$ was considered as statistical significance.

\section{Results}

Se increased plasma NO and decreased plasma vWF in Hcy-induced endothelial dysfunction rats

Hcy is a thiol-containing amino acid derived from L-methionine, one of the essential amino aids. The Hcy-induced endothelial dysfunction animal model was established by long term intragastric administration with L-methionine in rats. Se was added in the diet of Hcyinjected rats, and the concentration of plasma NO and vWF, two important biomarkers of endothelial injury were measured. As shown in Fig. 1, Hcy significantly reduced the level of plasma NO but increased the level of plasma vWF, which were significantly reversed by Se administration.

Se reversed Hcy-induced impairment of endothelium-dependent relaxation

To find out whether Se could prevent Hcy-induced endothelial dysfunction, relaxation in response to acetylcholine and sodium nitroprusside were examined on aortic rings. Acetylcholine caused a dose-dependent relaxation in precontracted aortic rings in all 3
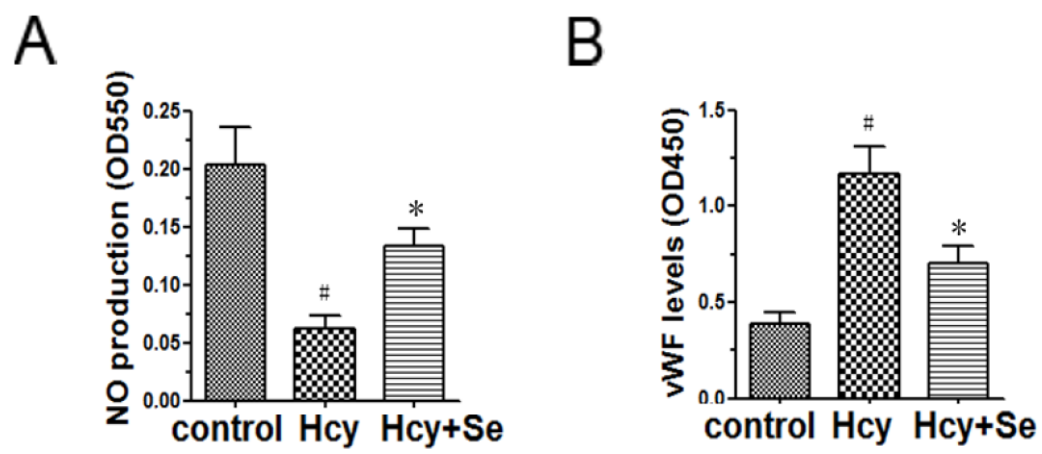

Fig. 1. The impacts of L-methionine and Se on biomarkers of endothelial injury in rats. (A) The impact of Lmethionine and Se on plasma NO. (B) The impact of L-methionine and Se on plasma vWF. The Hcy-induced endothelial dysfunction animal model was established by long-term intragastric administration with L-methionine in rats. Se was added in the treatment group. Plasma NO and vWF concentration were measured. The data are shown as mean \pm SD $(n=6)$. \# $\mathrm{p}<0.05$ versus control group, ${ }^{*} \mathrm{p}<0.05$ versus L-methionine administrated rats. 


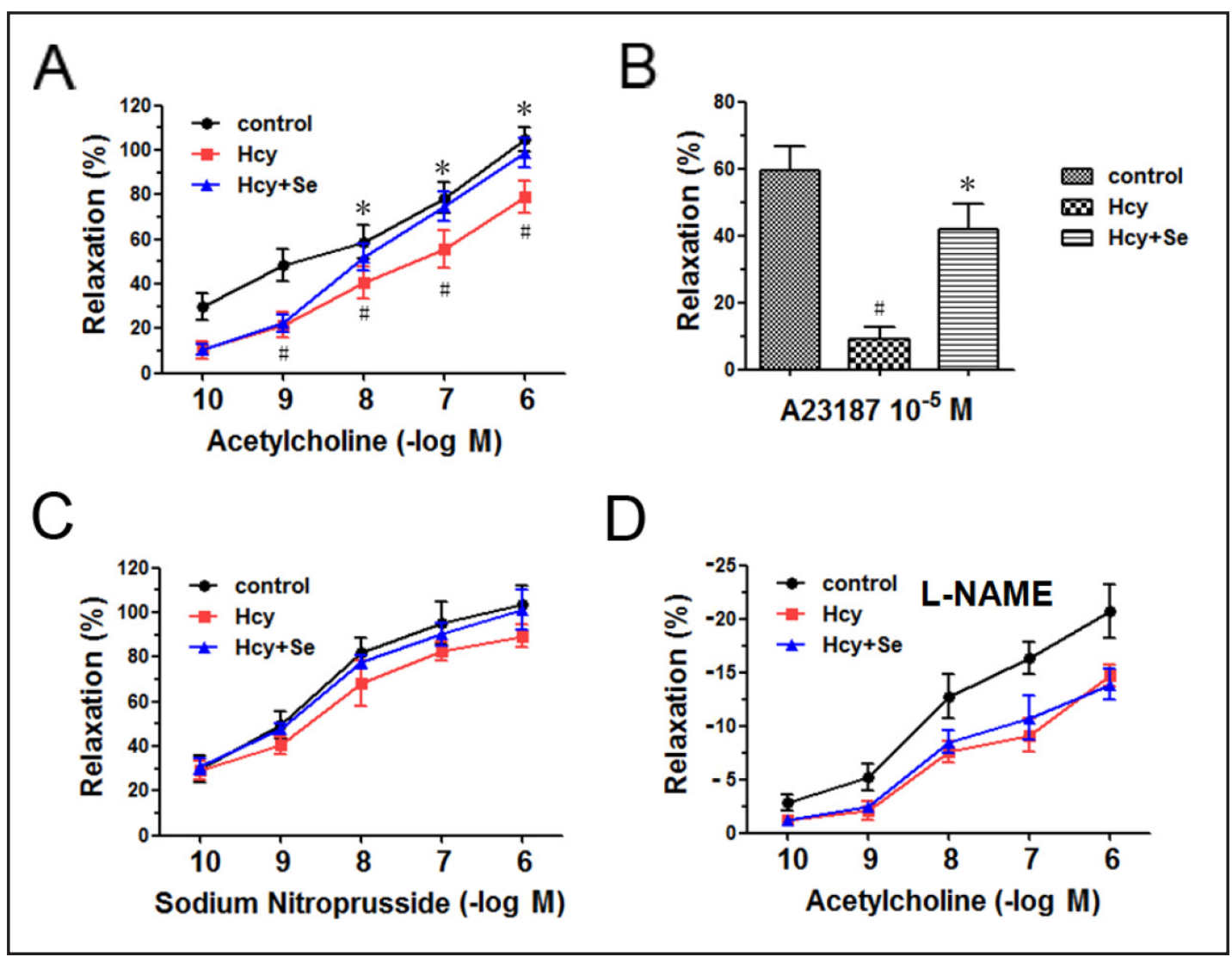

Fig. 2. Effects of L-methionine administration and Se on the rat aortic relaxation. (A) Relaxation was measured in response to the addition of acetylcholine. Thoracic aortic rings were precontracted with $\mathrm{KCl}$ and contracted with phenylephrine. Dose-response relaxation was measured for cumulative increments of $10^{-10}-10^{-6}$ M acetylcholine. (B) Relaxation was measured in response to the addition of $10^{-5} \mathrm{M}$ A23187. (C) Relaxation was measured in response to the addition of $10^{-10}-10^{-6} \mathrm{M}$ sodium nitroprusside. (D) The effect of L-NAME on relaxation in response to acetylcholine. Aortic rings were precontracted with $\mathrm{KCl}$, treated with $3 \times 10^{-5} \mathrm{M}$ L-NAME for $30 \mathrm{~min}$, and contracted with phenylephrine. Dose-response relaxation was measured for cumulative increments of acetylcholine. The data are shown as mean $\pm \operatorname{SD}(n=6)$. $\# p<0.05$ versus control group, $* \mathrm{p}<0.05$ versus Hcy group. Hcy, with only L-methionine administration; Hcy+Se, with both L-methionine and Se administration.

groups. Hcy significantly impaired the relaxation to acetylcholine, while Se attenuated Hcy-induced impairment of relaxation (Fig. 2A). Similar changes were also observed in the relaxation to A23187, a NOS activator (Fig. 2B). Endothelium independent relaxation produced by NO donor, sodium nitroprusside was similar between 3 groups (Fig. 2C). To define the contribution of NO in the Se-induced enhancement of endothelium dependent relaxation, Nnitro-L-arginine methylester hydrochloride (L-NAME), a specific NOS inhibitor, was added before the addition of acetylcholine. As shown in Fig. 2D, pretreatment of vessels with L-NAME abolished the relaxation response to acetylcholine in all 3 groups, there was no difference between the relaxation response of Hcy group and Hcy+Se group. These results suggested that Hcy impairs endothelium-dependent relaxation and Se reverse Hcy-induced impairment of relaxation. NO may participate in the protective role of Se.

Se attenuated the detrimental effects of Hcy on viability and migration of HUVECs

To explore the effect of Se on Hcy-induced endothelial cells dysfunction, we then examined the viability and migration of HUVECs under Hcy incubation with or without Se pretreatment. As shown in Fig. 3A, cellular viability of HUVECs was inhibited by incubation 


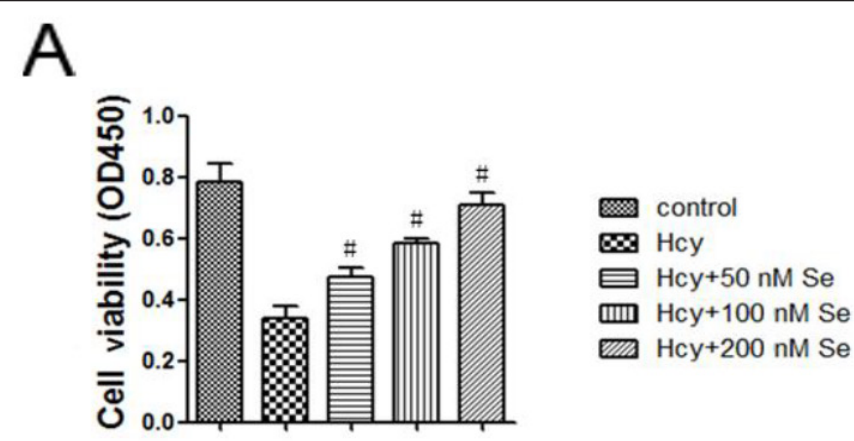

B
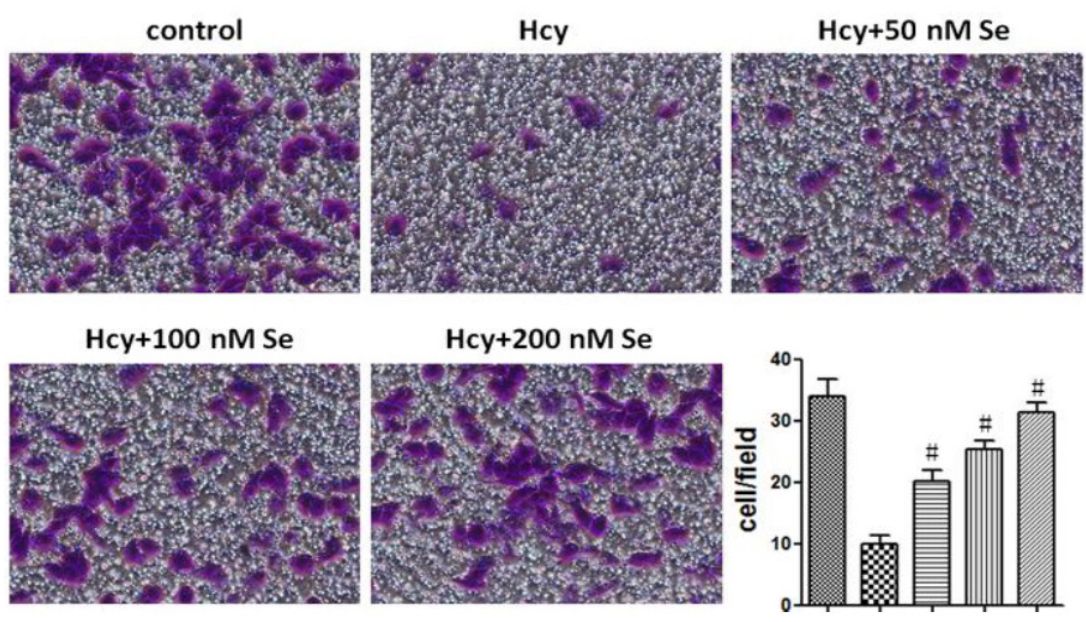

Fig. 3. Se rescued the impaired viability and migration of HUVECs induced by Hcy. (A) Se rescued the impaired cell viability induced by Hcy. (B) Se rescued the impaired cell migration induced by Hcy. HUVECs were pretreated with $0,50,100$, or $200 \mathrm{nM}$ Se for $12 \mathrm{~h}$, followed by incubation with $500 \mu \mathrm{M}$ Hcy for $24 \mathrm{~h}$. Otherwise cells treated with neither Hcy nor Se were used as control. Data are shown as means \pm SD. ( $n=3)$. \# P $<0.05$ versus Hcy group $(500 \mu \mathrm{M})$.

with $500 \mu \mathrm{M}$ Hcy for $24 \mathrm{~h}$ compared with the control, consisting with previous reports [16]. The detrimental effects of Hcy on HUVECs were attenuated by Se (50, 100 and $200 \mathrm{nM}$ ) pretreatment for $12 \mathrm{~h}$. Different concentrations of Se were added (50,100 and $200 \mathrm{nM}) 12 \mathrm{~h}$ before $500 \mu \mathrm{M}$ Hcy stimulation. Similarly, we found that Hcy-induced migration of HUVECs was rescued by Se in a dose-dependent manner (Fig. 3B). These results revealed that Se treatment reversed HUVECs viability and migration induced by Hcy.

\section{Se attenuated the Hcy-induced HUVECs apoptosis}

To test the effect of Se on Hcy-induced endothelial cells apoptosis, we detected the cell apoptosis rates and the expression of apoptosis-related protein. As demonstrated in Fig. 4A, Hcy treatment resulted in significant increase in HUVECs apoptosis. When cells were incubated with Se, the apoptosis were dramatically downregulated in a dose-dependent manner (Fig. 4A). The Caspase family members of cysteine proteases are key players in the programmed cell death, and Caspase- 3 has been proven to be the most important component of the Caspase family [17]. Therefore we determined the activity of Caspase- 3 in Hcy-treated HUVECs using a colorimetric activity assay. As shown in Fig. 4B, the activity of Caspase-3 displayed a similar change after Se was added into the culture media of HUVECs. Then the expression of cleaved Caspase-3, Bax, and Bcl-3 were examined by Western blotting. As expected, the level of cleaved Caspase-3 was obviously elevates after Hcy treatment. When the cells were pretreated with different concentrations of Se, the levels of cleaved Caspase- 3 was gradually dropped (Fig. 4C and D). Another cell apoptosis indicator, Bax, also demonstrated 


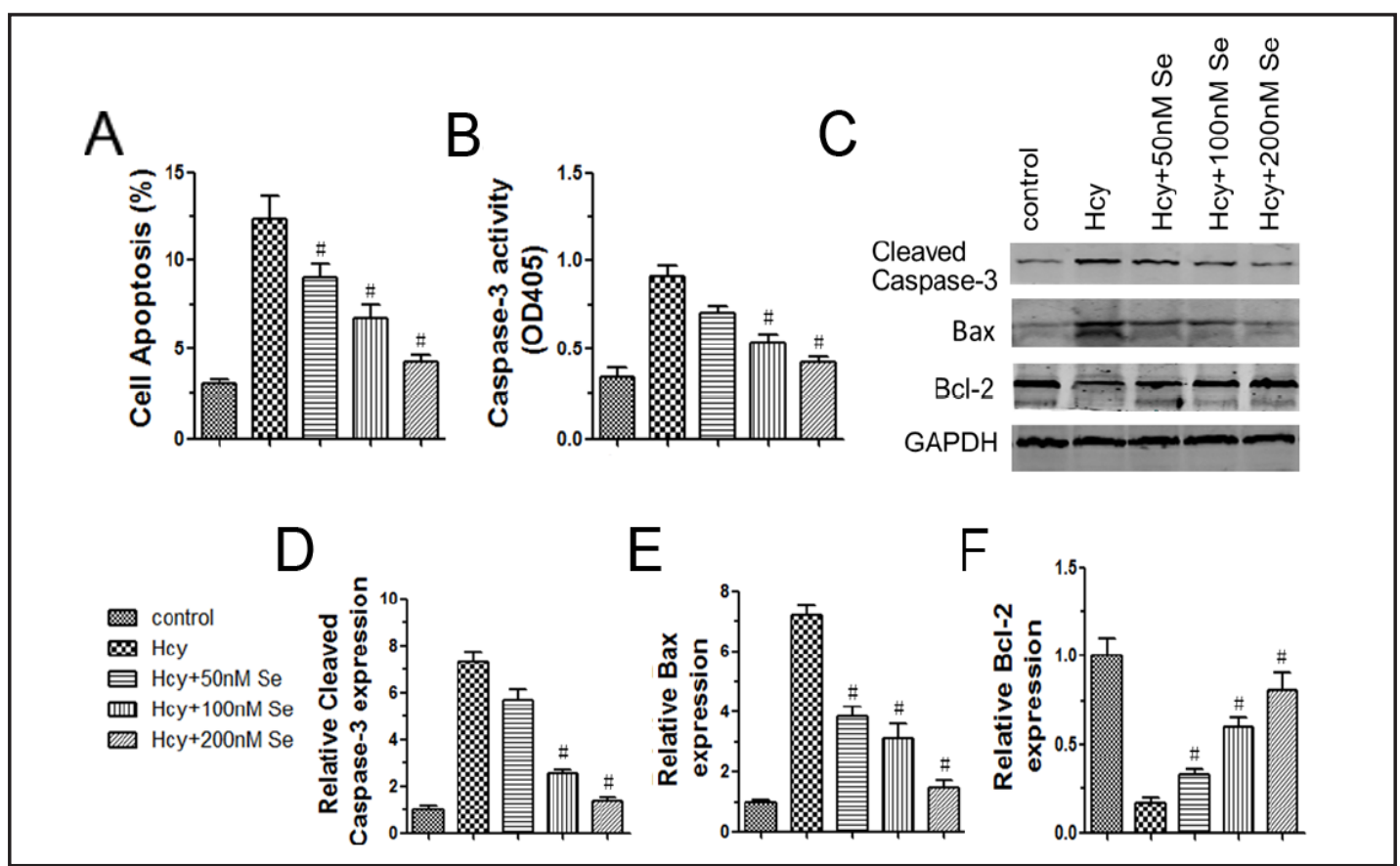

Fig. 4. Se inhibited the Hcy-induced apoptosis of HUVECs. (A) Se inhibited the Hcy-induced apoptosis of endothelial cells. (B) Se impaired the activity of Caspase-3 induced by Hcy. (C) Se regulated the expression of cleaved Caspase-3, Bax, and Bcl-2. (D) Quantitative densitometry data of cleaved Caspase-3 levels in different groups. (E) Quantitative densitometry data of Bax levels. (F) Quantitative densitometry data of Bcl-2 levels. Cells were pretreated with $0,50,100$, or $200 \mathrm{nM} \mathrm{Se}$ for $12 \mathrm{~h}$, followed by incubation with $500 \mu \mathrm{M} \mathrm{Hcy}$ for $24 \mathrm{~h}$. Otherwise cells treated with neither Hcy nor Se were used as control. Data are shown as means \pm SD. $(\mathrm{n}=3)$. \# $\mathrm{P}<0.05$ versus Hcy group $(500 \mu \mathrm{M})$.

a similar trend after Se was added (Fig. 4C and E). On the contrary, the expression of an anti-apoptotic indicator, Bcl-2 was decreased significantly after Hcy treatment. However, the decrease was reversed after Se was supplemented (Fig. 4C and F). The levels of cleaved Caspase-3, Bax, Bcl-2 was demonstrated in Fig. 4D, E, and F respectively.

\section{Se increased NO production and eNOS phosphorylation and expression in HUVECS}

To test whether Se could affect NO bioavailability under Hcy treatment, we investigated NO production in HUVECs. As shown in Fig. 5A, NO levels in the presence of Hcy showed a dose-dependent reduction with a significant induction at $500 \mu \mathrm{M}$ Hcy. Pretreatment with Se for $12 \mathrm{~h}$ attenuated the detrimental effect of Hcy on NO production (Fig. 5B). Cells were also pretreated with $50 \mathrm{nM}$ Se for indicated times before $500 \mu \mathrm{M}$ Hcy stimulation. NO levels in the presence of Se showed significant increases for 24 and 36 h pretreatment (Fig. 5C). Furthermore, Fig. 5D and $\mathrm{E}$ demonstrated that the down-regulation of protein and mRNA of eNOS expression induced by Hcy was rescued by Se pretreatment (50, 100, and $200 \mathrm{nM}$ ). The decrease of the ratio of phosphorylated/total eNOS induced by Hcy was rescued by Se pretreatment (100, and 200nM). (Fig. 5D, F). Similarly, this protective role of Se was played in a dose-dependent manner.

\section{Se rescued Hcy-induced dysfunction of HUVECs via AKT activation}

To find out the mechanism by which Se functions, we detected several pathways, such as AKT, MAPK, NFkB, to determine which one was involved. As shown in Fig. 6A and $B$, Hcy treatment significantly decreased the phosphorylation of AKT compared with the control. When HUVECs were pretreated with Se $(50,100$, and $200 \mathrm{nM})$, the decrease in the levels of phosphorylated AKT was upregulated significantly. Se reversed Hcy-induced dephosphorylation of AKT without altering the protein expression of total AKT. To determine 
A

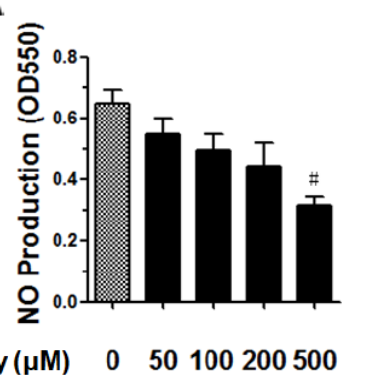

Hcy $(\mu \mathrm{M}) \quad 0 \quad 50100200500$

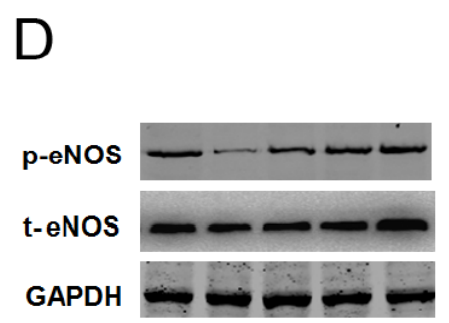

Hcy $(\mu \mathrm{M}) \quad 0 \quad 500500500500$ Se (nM) $00 \begin{array}{lllll}50 & 50 & 100\end{array}$

\section{B}

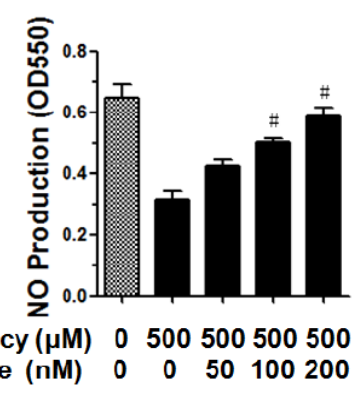

$\mathrm{E}$

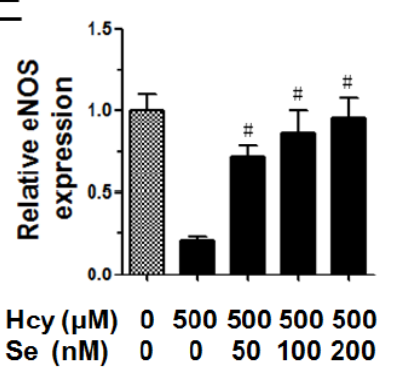

C

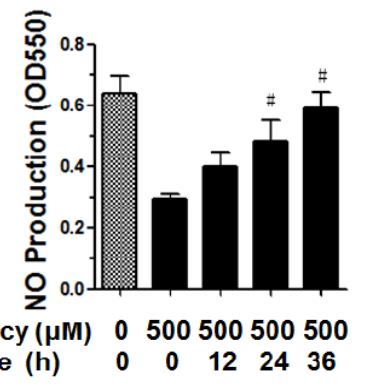

$\mathrm{F}$

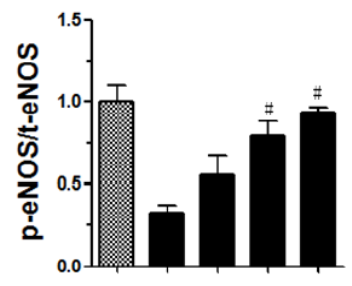

Hcy $(\mu \mathrm{M}) \quad 0500500500500$ Se (nM) $\quad 0 \quad 0 \quad 50100200$

Fig. 5. Se increased the impaired NO production, eNOS expression and phosphorylation induced by Hcy. (A) The effect of Hcy on NO production. HUVECs were treated with various concentrations of Hcy for 24 $h$, and the NO production was measured. (B) Se rescued the impaired NO production induced by Hcy in a dose-dependent manner. HUVECs were treated with various concentrations of Hcy for $24 \mathrm{~h}$ and were pretreated with various concentrations of Se for $12 \mathrm{~h}$, and the NO production was measured. (C) Se rescued the impaired NO production induced by Hcy in a time-dependent manner. HUVECs were treated with various concentrations of Hcy for $24 \mathrm{~h}$ and were pretreated with $50 \mathrm{nM} \mathrm{Se}$, at the indicated time points the NO production was measured. (D) Se rescued the impaired expression and phosphprylation of eNOS induced by Hcy. Cells were pretreated with $0,50,100$, or $200 \mathrm{nM}$ Se for $12 \mathrm{~h}$, followed by incubation with $500 \mu \mathrm{M}$ Hcy for $24 \mathrm{~h}$. Otherwise cells treated with neither Hcy nor Se were used as control. (E) Quantitative determination of eNOS mRNA expression by Realtime PCR. (F) Quantitative densitometry data of the ratio of phosphorylated/total eNOS levels. Data are shown as means \pm SD $(n=5)$. \# $P<0.05$ versus Se-free Hcy group (500 $\mu \mathrm{M}$ Hcy).

whether AKT plays roles in Se-induced eNOS phosphorylation and HUVECs' dysfunction, AKT inhibitor SH-5 was employed. As showed in Fig. 6 C to E, Hcy resulted in a significant inhibition of eNOS phosphorylation and expression, whereas $200 \mathrm{nM}$ Se pretreatment rescued these effects. When AKT inhibitor SH-5 was added, these effects were abolished, indicating that AKT was involved in the Se-induction of eNOS phosphorylation and expression. NO production was measured in the presence of SH-5. As shown in Fig. 6F, Se rescued the decreased levels of NO induced by Hcy treatment. When SH-5 was added, the protective role of Se was abolished. Similar results were also observed in cell viability, cell migration, and cell apoptosis (Fig. 6G-I). These findings indicated the involvement of AKT activation in the protective role of Se in the Hcy-induced dysfunction and apoptosis of HUVECs.

\section{Discussion}

CVD is the leading cause of death globally, much more people die annually from CVD than any other causes [18]. The World Health Organization (WHO) estimated that there will be about 20 million CVD deaths in 2015, accounting for $30 \%$ of all deaths worldwide [19]. A healthy diet, tobacco and alcohol control, and physical exercises are all included 
A

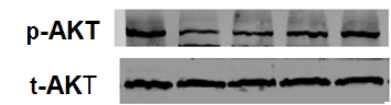

Hсу $(\mu \mathrm{M}) \quad 0 \quad 500500500500$ Se (nM) $\quad 0 \quad 00150 \quad 100 \quad 200$

D

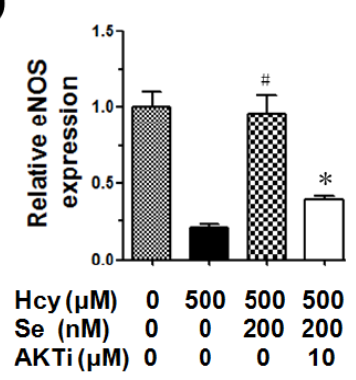

G

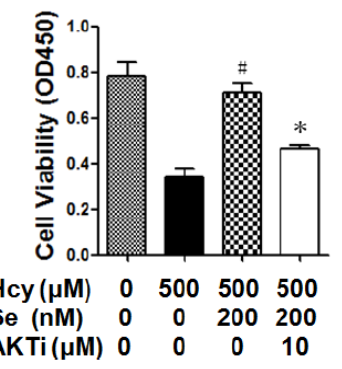

B

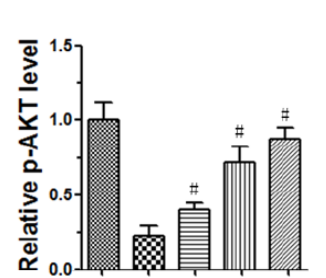

Hcy $(\mu M) \quad 0 \quad 500500500500$ Se (nM) $0 \quad 0 \quad 50100200$

E

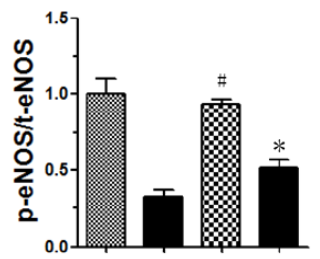

Hcy $(\mu \mathrm{M}) \quad 0 \quad 500500500$

$\begin{array}{llccc}\mathrm{Se}(\mathrm{nM}) & 0 & 0 & 200 & 200 \\ \mathrm{AKTi}(\mu \mathrm{M}) & 0 & 0 & 0 & 10\end{array}$

$\mathrm{H}$

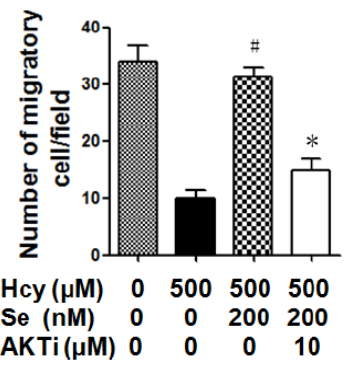

C

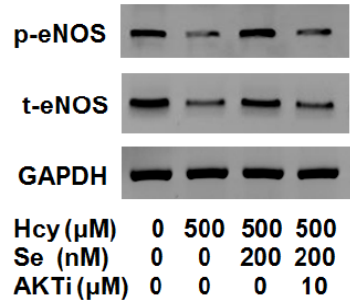

F

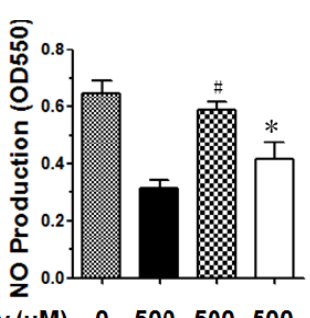

Hcy $(\mu \mathrm{M}) \quad 0 \quad 500500500$

Se (nM) $0 \quad 0 \quad 200200$

$\begin{array}{lllll}\operatorname{AKTi}(\mu \mathrm{M}) & 0 & 0 & 0 & 10\end{array}$

I

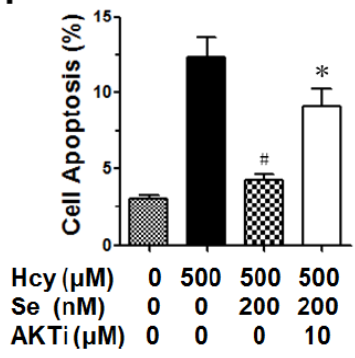

Fig. 6. Se rescued Hcy-induced dysfunction of HUVECs via AKT activation. (A) Se rescued the impaired phosphorylated AKT induced by Hcy. HUVECs were pretreated with 0, 50, 100, or $200 \mathrm{nM}$ Se for $12 \mathrm{~h}$, followed by incubation with $500 \mu \mathrm{M}$ Hcy for $24 \mathrm{~h}$. Otherwise cells treated with neither Hcy nor Se were used as control. (B) Quantitative densitometry data of phosphorylated AKT levels. (C) The rescue of expression and phosphorylation of eNOS by Se was AKT-dependent. HUVECs were pretreated with $200 \mathrm{nM}$ Se or $10 \mu \mathrm{M}$ SH-5 plus $200 \mathrm{nM}$ Se for $12 \mathrm{~h}$ before $500 \mu \mathrm{M}$ Hcy stimulation for $24 \mathrm{~h}$. The change of expression and phosphorylation of eNOS was detected by Western blot analysis. (D) Quantitative densitometry data of eNOS expression. (E) Quantitative densitometry data of the ratio of phosphorylated/total eNOS levels. (F) The Se-induced NO production was AKT-dependent. HUVECs were treated as described in (C) and NO production was measured. (G) The Se-induced cell viability was AKT-dependent. HUVECs were treated as described in (C) and cell viability was measured. (H) The Se-induced cell migration was AKT-dependent. (I) The decrease of cell apoptosis induced by Se was AKT-dependent. Data are shown as means \pm SD $(n=3)$. \# p $<0.05$ versus Hcy only group $(500 \mu \mathrm{M}){ }^{*} \mathrm{p}<0.05$ versus Hcy+Se group (500 $\mu \mathrm{M}$ Hcy+200 nM Se).

in the currently practiced measures to decrease the risk of CVD [20]. Therefore, exploring beneficial dietary supplements is of great importance for the prevention of CVD.

In the present study, we found that Se protected Hcy-induced endothelial dysfunction in vivo and in vitro. Se prevented Hcy-induced end othelia injury and impairment of endotheliumdependent relaxation. Se rescued the impaired viability and migration of HUVECs caused by Hcy treatment. In addition, Se inhibited the apoptosis of endothelial cells induced by Hcy, through downregulating of Caspase-3 activity, suppressing the expression of cleaved Caspase- 3 and Bax, and stimulating the expression of Bcl-2. Moreover, the downregulation of $\mathrm{NO}$ and eNOS caused by Hcy were rescued by Se treatment. Se increased the phosphorylation 


\section{Cellular Physiology Cell Physiol Biochem 2016;38:871-882 \\ \begin{tabular}{ll|l} 
and Biochemistry Published online: February 25, 2016 & $\begin{array}{l}\text { (c) } 2016 \text { The Author(s). Published by S. Karger AG, Basel } \\
\text { www.karger.com/cpb }\end{array}$ \\
\hline
\end{tabular} \\ Ren et al.: Selenium Protects Endothelial Function}

of AKT, and Se-induced phosphorylation and expression of eNOS, NO production, cell viability and migration were blocked by AKT inhibition.

Endothelium is a highly selective barrier and metabolically active organ, which plays crucial roles in the maintenance of vascular homeostasis [21]. Endothelial dysfunction (ED) is observed in the early stage of atherosclerosis [22], and is associated with the risk of atherosclerotic cardiovascular diseases [23]. Once the endothelial function deteriorates, the vascular homeostasis is disturbed, leading to reduced anti-oxidant and anti-inflammatory effects, increased vascular permeability to lipoproteins, increased expression of inflammatory cytokines and adhesion molecules, and subsequent development of atherosclerosis [24]. In our results, we demonstrated that Se increased the viability and migration of endothelial cells impaired by Hcy, while inhibiting the apoptosis induced by Hcy. The overall effect of Se on endothelial cells is protective, especially in the presence of Hcy.

Endothelial cells produce and release various kinds of factors, such as nitric oxide (NO), endothelium-derived hyperpolarizing factor (EDHF), prostacyclin, or endothelin-1 (ET-1), angiotensin II, thromboxane A2, prostaglandin $\mathrm{H} 2$ to keep the delicate balance between vasodilation and vasoconstriction [25]. Among these endothelial-derived vasodilators, NO is the most important one because of its key role in inhibiting platelet aggregation, inflammation, oxidative stress, vascular smooth muscle cell migration and proliferation, and leukocyte adhesion [26]. In our study, we showed that the impaired production of NO was rescued by Se. Endothelial nitric oxide synthetase (eNOS) is the direct enzyme responsible for NO production in endothelial cells $[27,28]$. Therefore we detected the effect of Se on the expression of eNOS. As expected, Se improved the expression of eNOS. The activity of eNOS is mainly regulated in post-translational level (phosphorylation). Ser-1177 in the reductase domain is one of the important phosphorylation sites in regulating eNOS activity [29], and phosphorylation of Ser-1177 is indispensible for eNOS activation. Our results showed that Se could not only rescue the expression of eNOS, but also increase the phosphorylation of eNOS at its Ser-1177 site. Although the total eNOS level was increased, the extent of increase in phosphorylated eNOS level was much greater. Therefore the overall trend of phosphorylated/total ratio is still elevated after Se pretreatment. Our results suggested that eNOS-NO signaling was responsible for the protective effects of Se on Hcy-induced endothelial dysfunction.

PI3K/AKT pathway plays important roles in vascular homeostasis, including regulation of cell survival, migration, and angiogenesis, glucose metabolism, and protein synthesis $[30,31]$. AKT stimulates eNOS phosphorylation at Ser-1177 and promotes subsequent NO production [32]. Inhibition of AKT activation by dominant negative AKT mutants prevents enhanced NO synthesis mediated by eNOS [33]. Other studies indicated the role of AKT in Hcy-induced cell cycle G1 arrest [34] and impaired endothelial function [35]. Given these background, we hypothesized that Se may protect the function of endothelial cells in an AKT-dependent manner. As expected, we found that Se-induced eNOS phosphorylation at Ser-1177 was abolished by AKT inhibitor SH-5, indicating that AKT activation participated the Se-induced phosphorylation of eNOS at Ser-1177, NO production, cell viability and migration. Collectively, we showed for the first time that Se reversed Hcy-induced inhibition of AKT phosphorylation at Ser-473 and eNOS phosphorylation at Ser-1177.

Se is a main ingredient of the glutathione peroxidase that protects cells from oxidative damage resulting from reactive oxygen species (ROS) [36]. The protective roles for Se in human health have been shown in recent studies [37]. Se deficiency has been reported in patients with different disorders including stroke, atherosclerosis, osteoarthritis, Alzheimer's diseases, hypothyroidism, and diabetes [38]. Many studies have indicated the benefits that Se may have on patients with hypertension and atherothrombotic diseases [39]. However, few studies have investigated the effect of Se on Hcy-induced dysfunction and apoptosis of endothelial cells. Hence, this study aimed to explore whether Se could relieve Hcy-induced effects on endothelial cells. Our results demonstrated that Se increased plasma NO levels and rescued the impaired endothelium-dependent relaxation to acetylcholine, but had no effect on sodium nitroprusside-induced endothelium-independent relaxation in rat aorta. Our 


\section{Cellular Physiology Cell Physiol Biochem 2016;38:871-882

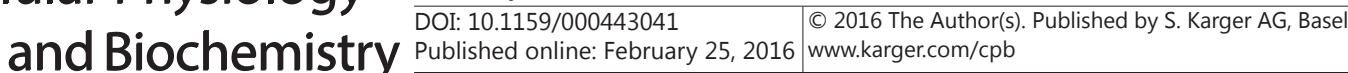 \\ Ren et al.: Selenium Protects Endothelial Function}

findings also suggested that Se has beneficial effects on endothelium and endothelial cells. We tested the effect of different concentrations of Se on Hcy-induced dysfunction of HUVECs in vitro, but did not detect the effect of Se alone on endothelial function. The reason is that Se alone did not show obvious influence on endothelium function in our animal experiments.

We performed permeability assays to test the endothelial permeability after Se treatment. HUVEC cells were seeded on collagen-coated PTFE membranes, and permeability was evaluated by FITC-dextran $40 \mathrm{kDa}$ passage. However, it seems that there was no significant difference between the Se-pretreatment group and control. The reason for that may be due to the size of the tracer, therefore we will use other FITC-dextran (such as 150 or $250 \mathrm{kDa}$ ) or other method to test the endothelial permeability in our future studies.

Taken together, for the first time our results demonstrated the role of Se on regulation of the function and apoptosis of HUVECs, enriching the current knowledge of Se, and providing important implications for the prevention of CVD in the future.

\section{Acknowledgements}

This work was supported by National Science \& Technology Pillar Program during the 12th Five-year Plan Period (2011BAI11B01).

\section{Disclosure Statement}

The authors state that they have no conflicts of interest.

\section{References}

1 Lee WW, Choi KC, Yum RW, Yu DS, Chair SY: Effectiveness of motivational interviewing on lifestyle modification and health outcomes of clients at risk or diagnosed with cardiovascular diseases: A systematic review. Int J Nurs Stud 2016;53:331-341.

2 Dugani S, Gaziano TA: 25 by 25: Achieving Global Reduction in Cardiovascular Mortality. Curr Cardiol Rep 2016;18:10.

3 Dubbs SB, Tewelde SZ: Cardiovascular Catastrophes in the Obstetric Population. Emerg Med Clin North Am 2015;33:483-500.

4 Ansar S, Koska J, Reaven PD: Postprandial hyperlipidemia, endothelial dysfunction and cardiovascular risk: focus on incretins. Cardiovasc Diabetol 2011;10:61.

5 Jufri NF, Mohamedali A, Avolio A, Baker MS: Mechanical stretch: physiological and pathological implications for human vascular endothelial cells. Vasc Cell 2015;7:8.

6 García-Ponce A, Citalán-Madrid AF, Velázquez-Avila M, Vargas-Robles H, Schnoor M: The role of actinbinding proteins in the control of endothelial barrier integrity. Thromb Haemost 2015;113:20-36.

7 Banecka-Majkutewicz Z, Sawuła W, Kadziński L, Węgrzyn A, Banecki B: Homocysteine, heat shock proteins, genistein and vitamins in ischemic stroke--pathogenic and therapeutic implications. Acta Biochim Pol 2012;59:495-499.

8 Baggott JE, Tamura T: Homocysteine, iron and cardiovascular disease: a hypothesis. Nutrients 2015;7:1108-1118.

9 Manolescu BN, Oprea E, Farcasanu IC, Berteanu M, Cercasov C: Homocysteine and vitamin therapy in stroke prevention and treatment: a review. Acta Biochim Pol 2010;57:467-477.

10 Jamaluddin MS, Yang X, Wang H: Hyperhomocysteinemia, DNA methylation and vascular disease. Clin Chem Lab Med 2007;45:1660-1666.

11 Almashhadany A, Shackebaei D, Van der Touw T, Jones GL, Suleiman MS, King N: Homocysteine Exposure Impairs Myocardial Resistance to Ischaemia Reperfusion and Oxidative Stress. Cell Physiol Biochem 2015;37:2265-2274.

12 Burk RF, Hill KE: Regulation of Selenium Metabolism and Transport. Annu Rev Nutr 2015;35:109-134.

13 Allingstrup M, Afshari A: Selenium supplementation for critically ill adults. Cochrane Database Syst Rev 2015;7:CD003703. 


\section{Cellular Physiology Cell Physiol Biochem 2016;38:871-882

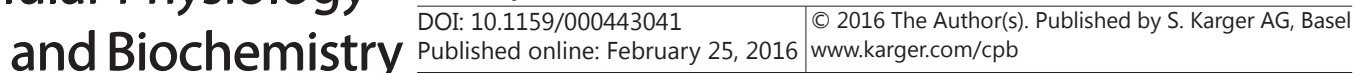 \\ Ren et al.: Selenium Protects Endothelial Function}

14 Ungvari Z, Pacher P, Rischa'k K: Dysfunction of nitric oxide mediation in isolated rat arterioles with methionine diet-induced hyperhomocysteinemia. Arterioscler Thromb Vasc Biol 1999;:1899-1904.

15 Ghajar CM, Blevins KS, Hughes CC, George SC, Putnam AJ: Mesenchymal stem cells enhance angiogenesis in mechanically viable prevascularized tissues via early matrix metalloproteinase upregulation. Tissue Eng 2006;12:2875-2888.

16 Zhou LJ, Mei YF, Wang LF, Jin H, Li SJ, Yin XS, Li WM: Homocysteine' s effect on tPA and PAI-1 gene expression in HUVEC. Zhongguo Ying Yong Sheng Li Xue Za Zhi 2004;20:363-366.

17 Yang B, Ye D, Wang Y: Caspase-3 as a therapeutic target for heart failure. Expert Opin Ther Targets 2013;17:255-263.

18 Alagona P Jr, Ahmad TA: Cardiovascular disease risk assessment and prevention: current guidelines and limitations. Med Clin North Am 2015;99:711-731.

19 Cosselman KE, Navas-Acien A, Kaufman JD: Environmental factors in cardiovascular disease. Nat Rev Cardiol 2015;12:627-642.

20 Ottawa (ON): Lifestyle Prescriptions: A Review of the Clinical Evidence. Canadian Agency for Drugs and Technologies in Health 2014.

21 Ivanov AN, Puchinyan DM, Norkin IA: Vascular endothelial Barrier Function. Usp Fiziol Nauk 2015;46:7296.

22 Peng J, Liu B, Ma QL, Luo XJ: Dysfunctional endothelial progenitor cells in cardiovascular diseases: role of NADPH oxidase. J Cardiovasc Pharmacol 2015;65:80-87.

23 Grimaldi V, Vietri MT, Schiano C, Picascia A, De Pascale MR, Fiorito C, Casamassimi A, Napoli C: Epigenetic reprogramming in atherosclerosis. Curr Atheroscler Rep 2015;17:476.

24 Sukmawati D, Tanaka R: Introduction to next generation of endothelial progenitor cell therapy: a promise in vascular medicine. Am J Transl Res 2015;7:411-421.

25 Husain K, Hernandez W, Ansari RA, Ferder L: Inflammation, oxidative stress and renin angiotensin system in atherosclerosis. World J Biol Chem 2015;6:209-217.

26 Zhao Y, Vanhoutte PM, Leung SW: Vascular nitric oxide: Beyond eNOS. J Pharmacol Sci 2015;129:83-94

27 Mount PF, Kemp BE, Power DA: Regulation of endothelial and myocardial NO synthesis by multi-site eNOS phosphorylation. J Mol Cell Cardiol 2007;42:271-279.

28 Xia M, Conley SM, Li G, Li PL, Boini KM: Inhibition of hyperhomocysteinemia-induced inflammasome activation and glomerular sclerosis by NLRP3 gene deletion. Cell Physiol Biochem 2014;34:829-841.

29 Peng X, Haldar S, Deshpande S, Irani K, Kass DA: Wall stiffness suppresses Akt/eNOS and cytoprotection in pulse-perfused endothelium. Hypertension 2003;41:378-381.

30 Shiojima I, Walsh K: Role of Akt signaling in vascular homeostasis and angiogenesis. Circ Res 2002;90:1243-1250.

31 Dimmeler S, Zeiher AM: Akt takes center stage in angiogenesis signaling. Circ Res 2000;86:4-5.

32 Heiss EH, Dirsch VM: Regulation of eNOS enzyme activity by posttranslational modification. Curr Pharm Des 2014;20:3503-3513.

33 Dimmeler S, Fleming I, Fisslthaler B, Hermann C, Busse R, Zeiher AM: Activation of nitric oxide synthase in endothelial cells by Akt-dependent phosphorylation. Nature 1999;399:601-605.

34 Zhang HS1, Cao EH, Qin JF: Homocysteine induces cell cycle G1 arrest in endothelial cells through the PI3K/ Akt/FOXO signaling pathway. Pharmacology 2005;74:57-64.

35 Yan TT, Li Q Zhang XH, Wu WK, Sun J, Li L, Zhang Q, Tan HM: Homocysteine impaired endothelial function through compromised vascular endothelial growth factor/Akt/endothelial nitric oxide synthase signalling. Clin Exp Pharmacol Physiol 2010;37:1071-1077.

36 Zhao J, Xing H, Liu C, Zhang Z, Xu S: Effect of Selenium Deficiency on Nitric Oxide and Heat Shock Proteins in Chicken Erythrocytes. Biol Trace Elem Res DOI:10.1007/s1201101505279.

37 Kipp AP, Strohm D, Brigelius-Flohé R, Schomburg L, Bechthold A, Leschik-Bonnet E, Heseker H; German Nutrition Society (DGE): Revised reference values for selenium intake. J Trace Elem Med Biol 2015;32:195199.

38 Kuruppu D, Hendrie HC, Yang L, Gao S: Selenium levels and hypertension: a systematic review of the literature. Public Health Nutr 2014;17:1342-1352.

39 Rees K, Hartley L, Day C, Flowers N, Clarke A, Stranges S: Selenium supplementation for the primary prevention of cardiovascular disease. Cochrane Database Syst Rev 2013;1:CD009671. 